\title{
Molecular pathogenesis of Neisseria gonorrhoeae
}

\section{Cynthia N. Cornelissen*}

Department of Microbiology and Immunology, Virginia Commonwealth University School of Medicine, Richmond, VA, USA

*Correspondence: cncornel@vcu.edu

This Research Topic is focused on the molecular mechanisms of pathogenesis employed by the obligate human pathogen, Neisseria gonorrhoeae. The 10 articles cover a range of topics, including updates on important virulence factors, vaccine development efforts, immune evasion by the gonococcus, and current models for assessing virulence, treatments, and prophylactic measures. In the chapter by Balthazar et al. (2011) the authors present a research article that describes a random mutagenesis screen for gonococcal mutants with reduced sensitivity to the bactericidal action of normal human serum. This screen led to the identification of lipooligosaccharide biosynthesis and modification genes, which appear to play important roles in serum resistance as well as resistance to cationic antimicrobial peptides. The review by Ramsey et al. (2011) describes the characteristics and contributions of a type 4 secretion system that is expressed by $80 \%$ of gonococcal isolates. This system secretes single-stranded DNA into the external milieu, resulting in free DNA in the environment. Subsequent transformation of gonococci within the population with this DNA does not require cell to cell contact and results in spread of genetic information within the population, enhancing antigenic diversity, and potentially spread of antibiotic resistance. Cornelissen and Hollander (2011) review the current knowledge on gonococcal TonB-dependent transporters and their contribution to iron acquisition. The transferrin-iron acquisition system is one of several transport systems that is comprised of a TonB-dependent transporter and an associated lipoprotein. These surface-exposed transferrin binding proteins are ubiquitously expressed by all gonococci, not subject to antigenic variation, and important for growth in vivo; therefore, these proteins are being pursued as potential vaccine antigens. Other TonB-dependent transporters are of unknown function, but their characteristics and potential functions are summarized. In the review by Falsetta et al. (2011) the authors describe the gonococcal requirements for biofilm formation on glass and on epithelial cell surfaces. Formation of a biofilm on epithelial cell surfaces is a survival strategy employed under stressful environmental conditions like those encountered in the human host. The gonococcal biofilm matrix is composed largely of DNA, and formation of the biofilm requires the release of membrane blebs, which contain DNA. The genes that are differentially regulated upon biofilm development are summarized, along with their individual contributions to the formation of robust biofilms. Gonococcal infections are characterized by an infiltration of PMNs to the site of infection; however, gonococci survive this assault and, in fact, can thrive within PMNs. Johnson and Criss (2011) review the mechanisms that allow the gonococcus to resist clearance by polymorphonuclear leukocytes (PMNs) including resistance to both oxidative and non-oxidative killing mechanisms. Gonorrhea infections do not elicit protective immunity, leaving persons who suffer from this disease susceptible to subsequent infections. In this issue,
Liu et al. (2011) summarize our current understanding of how the gonococcus subverts the immune system, and takes advantage of the immune privilege of the genital tract to foster an environment that is hospitable to this pathogen. Since infection does not elicit a protective response, a gonococcal vaccine has been sought for many years. Zhu et al. (2011) present a summary of gonococcal vaccine development efforts, including a previously unpublished study testing the efficacy of a viral replicon particle (VRP) expressing the PorB antigen. Several models have been developed with which to study gonococcal pathogenesis, and to examine the effectiveness of vaccination or treatment strategies. The review by Edwards and Butler (2011) describes an ex vivo model employing primary human cervical epithelial cells, and summarizes what is known about the pathobiology of gonococcal infection within the microenvironment of the lower human reproductive tract. The review focuses on adherence, invasion, host cell signaling events, and intracellular survival of the gonococcus within human epithelial cells of genital tract origin. Although the gonococcus is highly adapted to the human host, a mouse model of lower female genital tract colonization has been developed by Jerse and colleagues. In the review by Jerse et al. (2011) the characteristics of the estradiol-treated mouse model are summarized along with the various gonococcal mutants that are defective for colonization in this model. The utility of the mouse model for testing vaccines, antibiotics and microbicides, and the synergy between chlamydial and gonorrheal infections is also discussed. In the final chapter, Hobbs et al. (2011) summarize the results from human infection models, which have been used for decades to evaluate the molecular determinants to gonococcal virulence. This review also describes the strengths and weaknesses of the human model and its potential for testing vaccine candidates. This Frontiers Research Topic, dedicated to gonococcal pathogenesis, summarizes the progress that has been made over the past few years in this field, and highlights potential opportunities for future research. While much has been accomplished, much remains to be understood. With the incidence of gonococcal disease still unacceptably high, limited options for antimicrobial treatments, and no modes for immunoprotection, continued research in this field is critical. These reviews put into context and point the way toward key avenues of research, which could lead to decreases in incidence, novel treatment modalities, or long term protection against gonococcal disease.

\section{REFERENCES}

Balthazar, J. T., Gusa, A., Martin, L. E., Choudhury, B., Carlson, R., and Shafer, W. M. (2011). Lipooligosaccharide structure is an important determinant in the resistance of Neisseria gonorrhoeae to antimicrobial agents. Front. Microbiol. 2:30. doi: 10.3389/ fmicb.2011.00030

Cornelissen, C. N., and Hollander, A. (2011). TonB-dependent transporters expressed by Neisseria gonorrhoeae. Front. Microbiol. 2:117. doi: 10.3389/fmicb.2011.00117 
Edwards, J. L., and Butler, E. K. (2011). The pathobiology of Neisseria gonorrhoeae lower female genital tract infection. Front. Microbiol. 2:102. doi: 10.3389/ fmicb.2011.00102

Falsetta, M. L., Steichen, C. T., McEwan, A. G., Cho, C., Ketterer, M., Shao, J., Hunt, J., Jennings, M. P., and Apicella, M. A. (2011). The composition and metabolic phenotype of Neisseria gonorrhoeae biofilms. Front. Microbiol. 2:75. doi: 10.3389/ fmicb.2011.00075

Hobbs, M. M., Sparling, P. F., Cohen, M. S., Shafer, W. M., Deal, C. D., and Jerse, A. E. (2011). Experimental gonococcal infection in male volunteers: cumulative experience with Neisseria gonorrhoeae strains FA1090 and MS11mkC. Front. Microbiol. 2:123. doi: $10.3389 /$ fmicb. 2011.00123

Jerse, A. E., Wu, H., Packiam, M., Vonck, R. A., Begum, A. A., and Garvin, L. E. (2011). Estradiol-treated female mice as surrogate hosts for Neisseria gonorrhoeae genital tract infections. Front. Microbiol. 2:107. doi: 10.3389/fmicb.2011.00107

Johnson, M. B., and Criss, A. K. (2011). Resistance of Neisseria gonorrhoeae to neutrophils. Front. Microbiol. 2:77. doi: 10.3389/fmicb.2011.00077

Liu, Y., Feinen, B., and Russell, M. W. (2011). New concepts in immunity to Neisseria gonorrhoeae: innate responses and suppression of adaptive immunity favor the pathogen, not the host. Front. Microbiol. 2:52. doi: 10.3389/ fmicb.2011.00052

Ramsey, M. E., Woodhams, K. L., and Dillard, J. P. (2011). The gonococcal genetic island and type IV secretion in the pathogenic Neisseria. Front. Microbiol. 2:61. doi: 10.3389/ fmicb.2011.00061

Zhu, W., Chen, C. J., Thomas, C. E., Anderson, J. E., Jerse, A. E., and Sparling, P. F. (2011). Vaccines for gonorrhea: can we rise to the challenge? Front. Microbiol. 2:124. doi: 10.3389/fmicb.2011.00124

Received: 19 October 2011; accepted: 24 October 2011; published online: 15 November 2011. Citation: Cornelissen CN (2011) Molecular pathogenesis of Neisseria gonorrhoeae. Front. Microbio. 2:224. doi: 10.3389/fmicb.2011.00224

This article was submitted to Frontiers in Cellular and Infection Microbiology, a specialty of Frontiers in Microbiology.

Copyright (C) 2011 Cornelissen. This is an open-access article subject to a non-exclusive license between the authors and Frontiers Media SA, which permits use, distribution and reproduction in other forums, provided the original authors and source are credited and other Frontiers conditions are complied with. 